\title{
Neutron features at the UPM neutronics hall
}

\author{
Hector Rene Vega-Carrillo . Eduardo Gallego Alfredo Lorente Isabel P. Rubio Roberto Méndez
}

\author{
H I G H L I G H T S
}

- Neutron spectra and ambient dose equivalent, of a neutronics hall has been estimated.

- Using Monte Carlo methods a detailed model of the hall and the new Al-made measuring bench were used to estimate neutron spectra and $\mathrm{H}^{*}(10)$.

- Neutron spectra were measured using a Bonner sphere spectrometer along the measuring bench, neutron spectra were used to estimate the $\mathrm{H}^{*}(10)$ and to calculate the total fluence rate.

- H*(10) was also measured using the neutron area monitor LB 6411 and compared with values obtained in the Monte Carlo calculations.

- Total fluence rate was fitted to the semiempirical model.

\begin{abstract}
A B S T R A C T
The neutronics hall of the Nuclear Engineering Department at the Polytechnical University of Madrid has been characterized. The neutron spectra and the ambient dose equivalent produced by an ${ }^{241} \mathrm{AmBe}$ source were measured at various source-to-detector distances on the new bench. Using Monte Carlo methods a detailed model of the neutronics hall was designed, and neutron spectra and the ambient dose equivalent were calculated at the same locations where measurements were carried out. A good agreement between measured and calculated values was found.
\end{abstract}

\section{Introduction}

For personal and ambient radiation survey it is necessary to use reliable measuring techniques. When radiation field are neutrons, it is essential to measure the neutron spectrum because the biological effects strongly depend on neutron energy (IshakBoushaki et al., 2012), in this aim recently new neutron spectrometers have been developed (Selwood and Monk, 2012; GómezRos, et al., 2011; Gómez-Ros et al., 2010).

Eventually neutron measuring devices must be calibrated on a proper facility; the Nuclear Engineering Department of the Polytechnical University of Madrid (DIN-UPM) has replaced the old Al-made bench (Gallego et al., 2004) for an improved version made of steel with better structural stability. In the new bench any measuring device can be automatically located with respect to the ${ }^{241} \mathrm{AmBe}$ source within $0.1 \mathrm{~mm}$ precision along horizontal and vertical directions. Using a webcam and a PC remote desk the detector electronics and the bench performance can be controlled through the Internet protocol (TCP/IP). This feature is important when a neutron monitor is calibrated, and it is required to do measurements at different source-to-detector distances for large counting times.

The neutronics hall at DIN-UPM is utilized to calibrate neutron measuring instruments and to irradiate materials with neutrons. Nowadays the bench has one ${ }^{241} \mathrm{AmBe}$ source; in the near future there are plans to have two additional ISO-recommended sources, bare ${ }^{252} \mathrm{Cf}$ and $\mathrm{D}_{2} \mathrm{O}$ moderated ${ }^{252} \mathrm{Cf}$ (ISO, 1989).

The response of a radiation monitor is a unique feature of the type of device, depending on characteristics of the calibrating neutron source, like the neutron spectrum and the dose-equivalent rate, and should not be a function of the experimental procedures employed neither the features of the calibration facility (ISO, 2000). In order to fulfill those conditions, it is necessary to take into account the attenuation and scattering of neutrons in air, as well as the neutron scattering in the ceiling, floor and walls of the 
calibration hall that shape the neutron field to be different from the "free-field" condition required for calibration.

The aim of this work is to characterize the neutron field of an ${ }^{241} \mathrm{AmBe}$ source measuring the neutron spectra, and the ambient dose equivalent at different source-to-detector distances on the new bench. Characterization was also carried out by Monte Carlo methods where, hall, source and bench detailed models were used.

\section{Materials and methods}

\subsection{Measurements}

To calibrate area-survey instruments for neutrons, the isotopic neutron sources recommended by the ISO are ${ }^{241} \mathrm{AmBe},{ }^{241} \mathrm{AmB}$, ${ }^{252} \mathrm{Cf}$, and ${ }^{252} \mathrm{Cf} / \mathrm{D}_{2} \mathrm{O}$ (ISO, 1989). At any calibration room the neutron spectrum, $\Phi_{E}(E)$, the total neutron fluence, $\phi$, and the ambient dose equivalent, $H^{*}(10)$, must be determined. The $H^{*}(10)$ is the dose equivalent that would be produced by an aligned and expanded radiation field in the ICRU sphere at a $10 \mathrm{~mm}$ depth.

In this work, the neutron spectra produced by a $111 \pm 10 \% \mathrm{GBq}$ ${ }^{241} \mathrm{AmBe}$ source were measured at $80,100,115,130,150$ and $180 \mathrm{~cm}$ on the bench with a Bonner sphere spectrometer with a $0.4 \varnothing \times 0.4 \mathrm{~cm}^{2}{ }^{6} \mathrm{Lil}(\mathrm{Eu})$ scintillator, BSS $/{ }^{6} \mathrm{Lil}(\mathrm{Eu})$.

Bonner sphere spectrometer with 0 (bare ${ }^{6} \mathrm{LiI}$ (Eu) scintillator), $5.08,7.62,12.70,20.32,25.40$, and $30.48 \mathrm{~cm}$-diameter polyethylene spheres were utilized (BSS $\left./{ }^{6} \mathrm{LiI}\right)$. From here on, these will be named $0,2,3,5,8,10$, and 12 in-diameter spheres respectively. This type of spectrometer has been widely used to determine the neutron spectra of different sources (Bedogni, 2011; IshakBoushaki et al., 2012; Viererbl et al., 2012), and different thermal neutron detectors (Bedogni et al., 2010; Ishak-Boushaki et al., 2012; Viererbl et al., 2012). With the BUNKIUT code (Lowry and Johnson, 1984) and the UTA4 response matrix (Hertel and Davidson, 1985), the count rates of Bonner spheres were used to unfold the neutron spectra, $\Phi_{E}(E)$.

In Fig. 1 is shown the new bench with the 10 in-diameter sphere. The bench is installed in a $16 \times 9 \times 8 \mathrm{~m}^{3}$ neutronics hall; the source is safely transported from its storage to the bench using a pneumatic system (Gallego et al., 2004).

For each position in the bench the neutron spectrum was measured with the $\mathrm{BSS} /{ }^{6} \mathrm{LiI}(\mathrm{Eu})$. The spectra were used to calculate the total fluence by using Eq. (1).

$\phi=\int_{E} \Phi_{E}(E) d E$

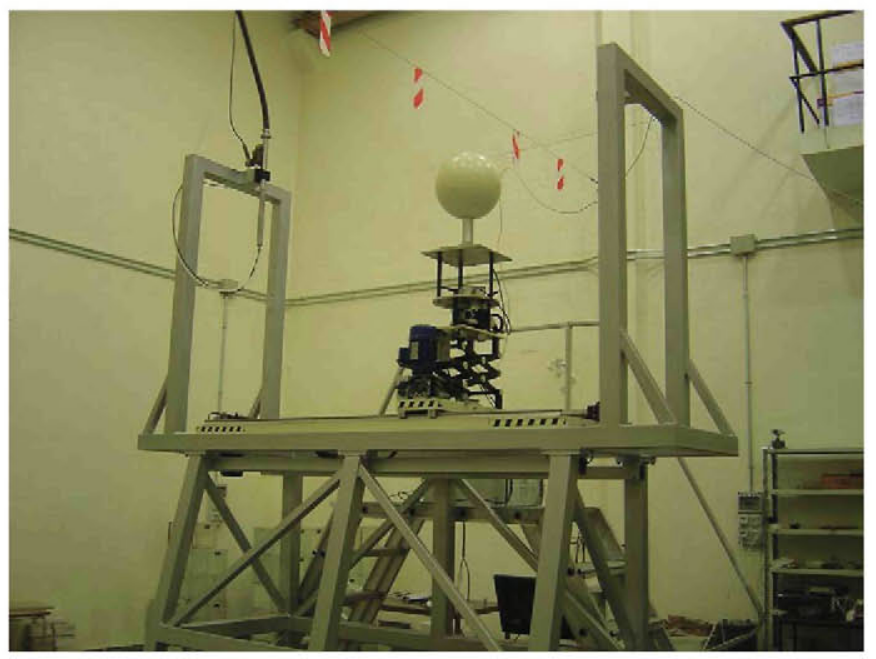

Fig. 1. Calibration bench. here, $\Phi_{E}(E)$ is the neutron spectrum in $\mathrm{cm}^{-2} \mathrm{MeV}^{-1}$ and $\phi$ is the total neutron fluence in $\mathrm{cm}^{-2}$.

Also the neutron spectra were used to calculate the ambient dose equivalent, $H^{*}(10)$, in each position on the bench using Eq. (2).

$H^{*}(10)=\int_{E} h_{\Phi}^{*}(E) \Phi_{E}(E) d E$

In Eq. (2), $h_{\Phi}^{*}(E)$ are the fluence-to-ambient dose equivalent coefficients, in pSv cm${ }^{2}$, from the ICRP 74 (ICRP, 1996) or the response of the neutron area monitor LB 6411 (Burgkhardt et al., 1997).

The total fluence values, in function of the source-to-detector distance, were fitted to the semi-empirical model (Hunt and Kluge, 1985; Eisenhauer et al., 1985) shown in Eq. (3).

$\phi(r)=\frac{B f(\theta)}{4 \pi r^{2}}\left\{\operatorname{Exp}[-\Sigma r]+A^{\prime} r+s^{\prime} r^{2}\right\}$

here, $B$ is the source strength in $s^{-1}, f(\theta)$ is the source anisotropy factor, $r$ is the distance between the center of the source and the reference point, in $\mathrm{cm}, \Sigma$ is the air linear attenuation coefficient for neutrons, in $\mathrm{cm}^{-1}, A^{\prime}$ and $s^{\prime}$ are proportionality constants in $\mathrm{cm}^{-1}$ and $\mathrm{cm}^{-2}$ respectively. Fitting was carried out using the weighted least-squares method (Vega-Carrillo, 1989), where the weighting factor was the reciprocal of the neutron fluence variance.

With the neutron area monitor Berthold LB 6411 (Burgkhardt et al., 1997) the $H^{*}(10)$ values were measured at the same locations on the bench where the neutron spectra were measured. These values were compared with those obtained through Eq. (2).

\subsection{Monte Carlo calculations}

With the MCNP5 code (X-5 Monte Carlo Team, 2005) a detailed model of the neutronics hall was designed, including the source's cladding, its support and the bench. In Fig. 2 the bench model is shown.

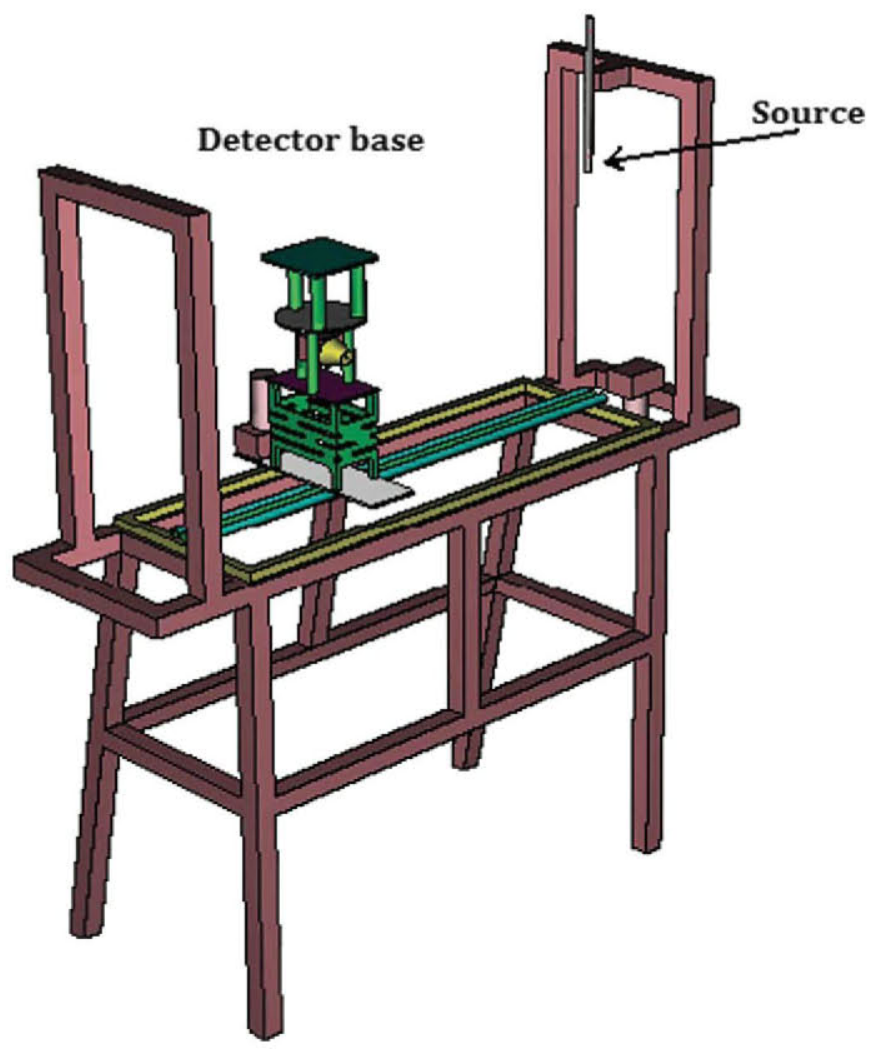

Fig. 2. Monte Carlo model of the new bench. 
In the Monte Carlo calculations, the source term for ${ }^{241} \mathrm{AmBe}$ was obtained from literature (ISO, 1989). Neutrons were isotropically transported from the cell utilized to model the source region; this cell was filled with the mixture of ${ }^{241} \mathrm{Am}$ and $\mathrm{Be}$.

Neutron spectra, and $H^{*}(10)$ were estimated at the same locations where measurements were carried out. In the calculations $1 E(7)$ histories were used allowing to have uncertainities less than $2 \%$.

\section{Results}

In Fig. 3 the lethargy spectra, in function of source-to-detector distance measured with the BSS, are shown.

In Fig. 3 it can be noticed that peak between 0.1 and $10 \mathrm{MeV}$ decreases as the source-to-detector distances increases while epithermal and thermal neutrons, are practically constant regardless to the source-to-detector distances due to the room-return effect characterized by the calibration hall dimensions (VegaCarrillo et al., 2007a, 2007b).

In Fig. 4, the calculated and measured neutron spectrum at $80 \mathrm{~cm}$ is shown.

In Fig. 5 the measured and calculated spectra at $180 \mathrm{~cm}$ are shown.

In Figs. 4 and 5 the agreement between the calculated and the measured spectrum can be noticed.

The total neutron fluence rate, obtained through the neutron spectrum using Eq. (1), in function to source-to-detector distance,

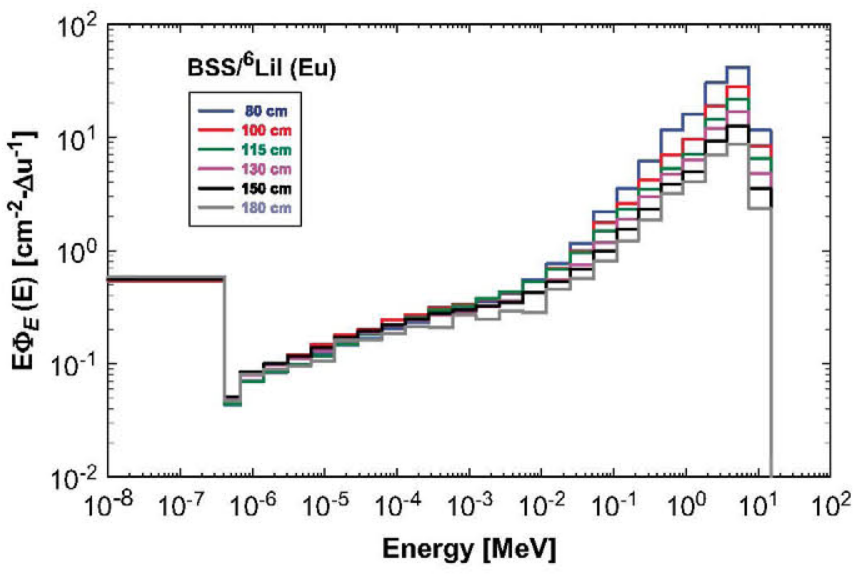

Fig. 3. Neutron spectra in function of the source-to-detector distance measured with the BSS $/{ }^{6}$ Lil.

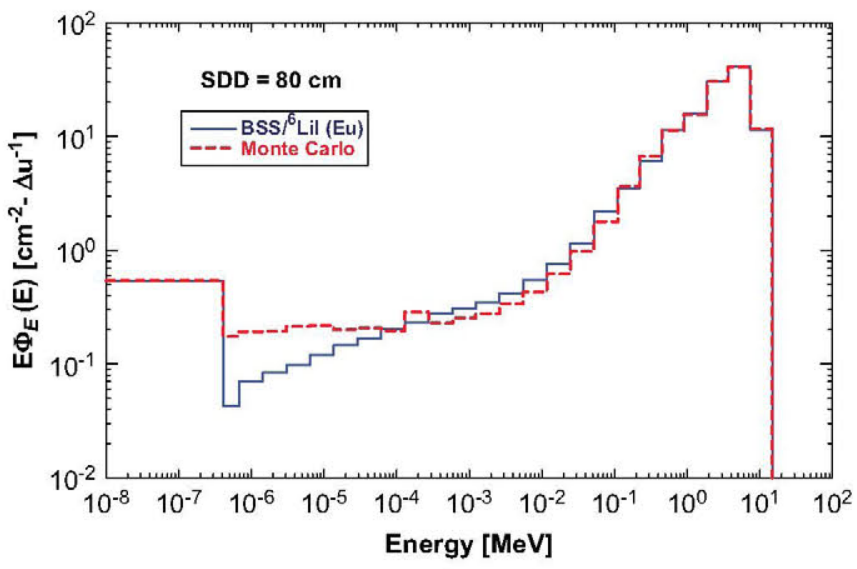

Fig. 4. Experimental and calculated spectra at $80 \mathrm{~cm}$.

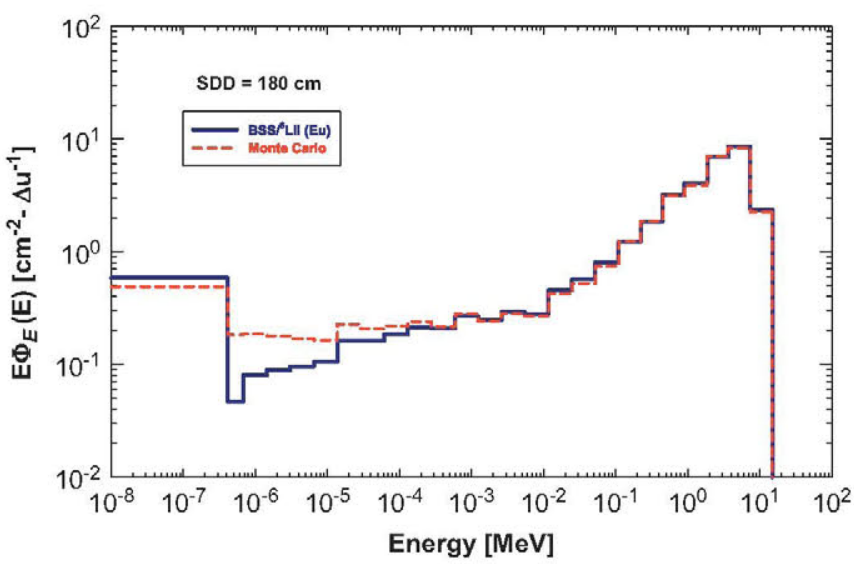

Fig. 5. Experimental and calculated spectra at $180 \mathrm{~cm}$.

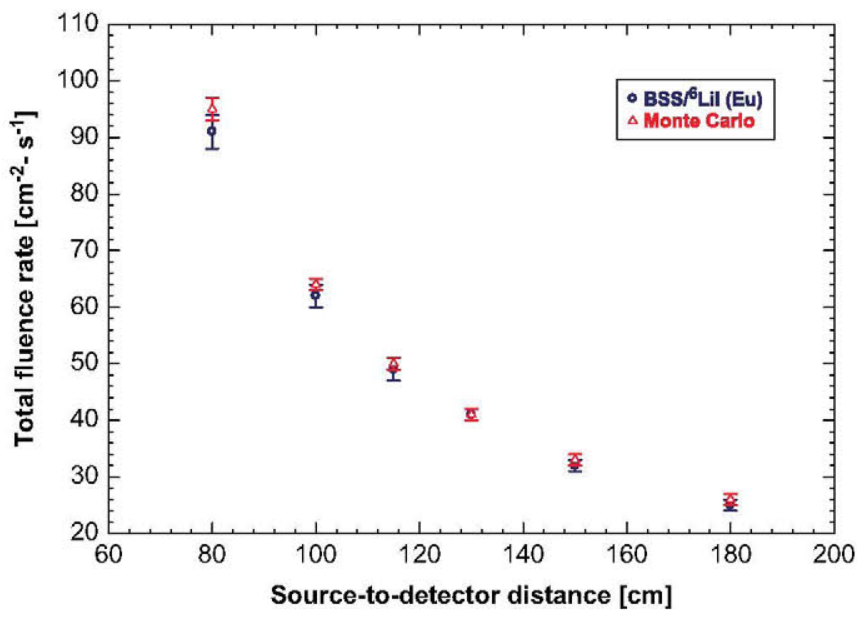

Fig. 6. Experimental and calculated total fluence rate in function of the source-todetector distances.

is shown in Fig. 6; here is also included the total fluence rate obtained by using the Monte Carlo spectra that was multiplied by the source strength. The source strength was calculated applying decay correction to source emissivity given by the manufacturer.

In Fig. 7 is the $H^{*}(10)$ values measured with the neutron area monitor LB 6411, in this figure the $H^{*}(10)$ values calculated through Eq. (2), using the spectra measured with the BSS $/{ }^{6} \mathrm{Lil}(\mathrm{Eu})$ and the fluence-to-dose coefficients from ICRP 74 (ICRP, 1996) (BSS (ICRP 74)), and the LB 6411 response function (Burgkhardt et al., 1997) (BSS (LB 6411)) are also included.

In Figs. 6 and 7 it can be also noticed that there is a good agreement between the calculated and measured values of $\phi(r)$ and $H^{*}(10)$.

Measured total fluence rates, in function of the source-todetector distance, were fitted to the semi-empirical model. The comparison between measured values and the fitted semiempirical model are shown in Fig. 8; the $\chi^{2}$ value was 0.0011 .

In the fitting process several parameters were calculated, one of these was the neutron source strength being $B=(5.83 \pm$ $0.57) \times 10^{6} \mathrm{~s}^{-1}$, that was calculated using a value of $1.04 \pm 0.01$ for the source anisotropy factor measured earlier that is in agreement to values reported for this type of sources (Bardell et al., 1998). The $B$-value is in agreement to the source strength reported by the source manufacturer, after doing the proper decay corrections. 


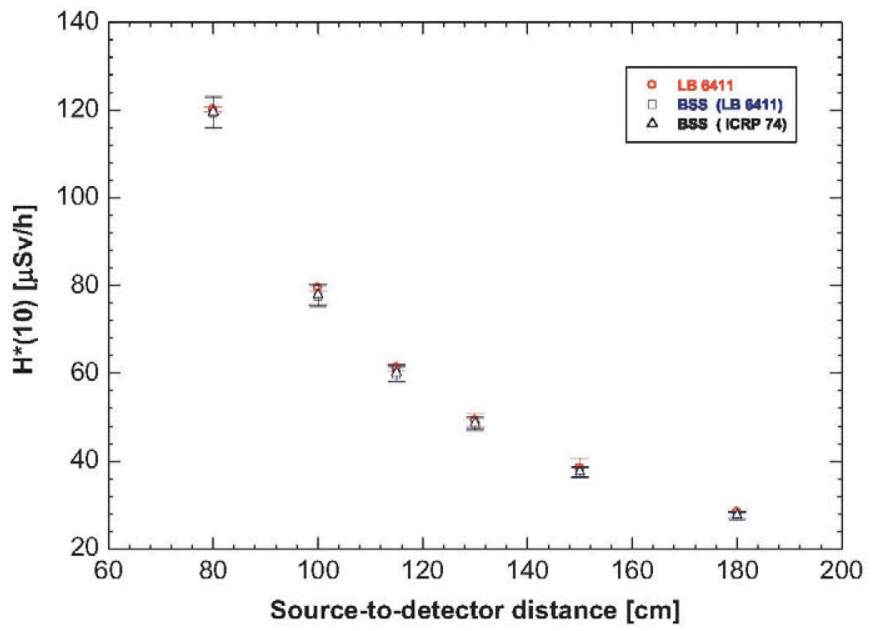

Fig. 7. Experimental and calculated $H^{*}(10)$ values on the bench.

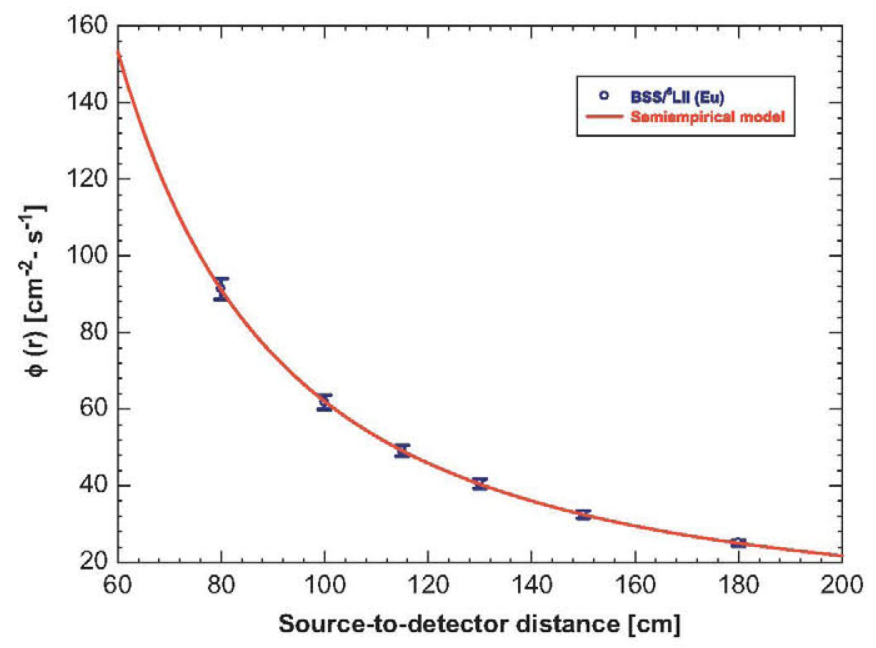

Fig. 8. Measured total neutron fluence and semiempirical model fit.

\section{Discussion and conclusion}

Neutron field produced by an ${ }^{241} \mathrm{AmBe}$ on the new bench from the neutronics hall of the DIN-UPM has been featured. Here, the neutron spectra and the $H^{*}(10)$ were measured in function of the source-to-detector distance on the new bench. Neutron spectra were measured with a Bonner sphere spectrometer, while $H^{*}(10)$ were measured with a monitor area LB 6411.

The characterization was also performed with the MCNP5 code using a detailed model of the hall. The results are alike to those obtained earlier (Gallego et al., 2004), therefore the change of Almade bench to a new and improved one made of steel do not introduce significant modifications.

The presence of the air, bench, and calibration hall walls shapes the neutron spectrum as well as the integral quantities, like $\phi$ and $H^{*}(10)$, resulting in a neutron field that is different from the desired "free field", required to calibrate neutron monitors (ISO, 2000).

Neutron spectra obtained in function of the source-to-detector distance on the bench show the presence of epithermal and thermal neutrons mainly produced when the source's neutrons collide with the ceiling, floor and walls of the hall returning back to the room after being moderated and thermalized (Eisenhauer et al., 1985; Vega-Carrillo et al., 2007a, 2007b).
As the distance with respect to the source is increased, the air in-and-out-scattering of neutrons are enhanced, and total fluence diverges from the $1 / r^{2}$ rule (Eisenhauer et al., 1895). Also, as the distance is increased the neutron spectra, in the region from 0.1 to $10 \mathrm{MeV}$, are decreased, while epithermal and thermal neutrons remain practically constant, due to the room-return effect (VegaCarrillo et al., 2007a, 2007b).

The $H^{*}(10)$, in function of the source-to-detector distance, does not follow the $1 / r^{2}$ rule due to the changes in the $\Phi_{E}(E)$ and due to the response function of neutron area monitor (Hunt and Kluge, 1985).

A good agreement between measured and calculated spectra was observed; the same was also noticed when total neutron fluence rate and $H^{*}(10)$ were compared, validating the Monte Carlo model of this facility.

Measured total neutron fluence rates in function of the sourceto-detector distances on the bench were fitted to the semiempirical model, from this process the parameter related to the ${ }^{241} \mathrm{AmBe}$ source strength was obtained being statistically equal to their actual value.

\section{Acknowledgments}

This work was performed as part of the specific agreement between CIEMAT and UPM on neutron calibration.

\section{References}

Bardell, A.G., Burke, M., Hunt, J.B., Tagziria, H., Thomas, D.J., 1998. Anisotropy of emission from radionuclide neutron sources. National Physical Laboratory Report CIRM 24.

Bedogni, R., 2011. Neutron spectrometry with Bonner spheres for area monitoring in particle accelerators. Radiat. Prot. Dosim. 146, 383-394.

Bedogni, R., Ferrari, P., Gualdrini, G., Esposito, A., 2010. Design and experimental validation of a Bonner sphere spectrometer based on Dysprosium activation foils. Radiat. Meas. 45, 1201-1204.

Burgkhardt, B., Fieg, G., Klett, A., Plewnia, A., Siebert, B.R.L., 1997. The neutron fluence and $H^{*}(10)$ response of the new LB 6411 remcounter. Radiat. Prot. Dosim. 70, 361-364.

Eisenhauer, C.M., Hunt, J.B., Schwartz, R.B., 1985. Calibration techniques for neutron personal dosimetry. Radiat. Prot. Dosim. 10, 43-57.

Gallego, E., Lorente, A., Vega-Carrillo, H.R., 2004. Characteristics of the neutron field of the facility at DIN-UPM. Radiat. Prot. Dosim. 110, 73-79.

Gómez-Ros, J.M., Bedogni, R., Moraleda, M., Romero, A., Delgado, A., Esposito, A., 2010. Design and validation of a single sphere multi-detector neutron spectrometer based on LiF:Mg,Cu,P thermoluminescent dosemeters. Radiat. Meas. $45,1220-1223$.

Gómez-Ros, J.M., Bedogni, R., Palermo, I., Esposito, A., Delgado, A., Angelone, M., Pillon, M., 2011. Design and validation of a photon insensitive multidetector neutron spectrometer based on Dysprosium activation foils. Radiat. Meas. 46 , $1712-1715$.

Hertel, N.E., Davidson, J.W., 1985. The response of Bonner spheres to neutrons from thermal to $17.3 \mathrm{MeV}$. Nucl. Instrum. Methods Phys. Res. A 238, 509-516.

Hunt, J.B., Kluge, H., 1985. Different relative response as a function of neutron energy of a number of neutron dose equivalent rate meters of the same type and a possible explanation. Radiat. Prot. Dosim. 12, 29-32.

ICRP, 1996. Conversion coefficients for use in radiological protection against external radiation. ICRP Publication 74. Annals of the ICRP, vol. 26, p. 199.

Ishak-Boushaki, G.M., Boukeffoussa, K., Idiri, Z., Allab, M., 2012. Thick activation detectors for neutron spectrometry using different unfolding methods: sensitivity analysis and dose calculation. Appl. Radiat. Isot. 70, 515-519.

ISO, 1989. Neutron reference radiations for calibrating neutron-measuring devices used for radiation protection purposes and for determining their response as a function of neutron energy. International Organization for Standarization 8529 .

ISO, 2000. Reference neutron radiations - calibration fundamentals of radiation protection devices related to the basic quantities characterizing the radiation field, Part 2. International Organization for Standarization 8529-2.

Lowry, K.A., Johnson, T.L., 1984. Modification to Iterative Recursion Unfolding Algorithms and Computer Codes to Find More Appropriate Neutron Spectra Naval Research Laboratory Report 5340 Washington, DC.

Selwood, M.J., Monk, S.D., 2012. Development of a portable isotropic neutron spectrometer. Appl. Radiat. Isot. http://dx.doi.org/10.1016/j.apradiso.2011. 12.010 . 
Vega-Carrillo, H.R., 1989. Least squares for different experimental data. Rev. Mex. Fis. $35,597-602$.

Vega-Carrillo, H.R., Manzanares, E., Iñiguez, M.P., Gallego, E., Lorente, A., 2007a. Study of room-return neutrons. Radiat. Meas. 42, 413-419.

Vega-Carrillo, H.R., Manzanares, E., Iñiguez, M.P., Gallego, E., Lorente, A., 2007b. Spectrum of isotopic neutron sources inside concrete walls spherical cavities. Radiat. Meas. 42, 1373-1379.
Viererbl, L., Klupák, V., Lahodová, Z., Marek, M., 2012. Comparison of neutron spectrum measurement methods used for the epithermal beam of the LVR-15 research reactor. Appl. Radiat. Isot. http://dx.doi.org/10.1016/j.apradiso. 2011.11.032.

X-5 Monte Carlo Team, 2005. MCNP A General Monte Carlo N-Particle Transport Code Version 5. Los Alamos National Laboratory Report LA-UR-03-1987. 